\title{
High-Gain Textile Antenna Array System for Off-Body Communication
}

\author{
Maria Lucia Scarpello, Luigi Vallozzi, Hendrik Rogier, and Dries Vande Ginste \\ Electromagnetics Group, Department of Information Technology (INTEC), Ghent University, Sint-Pietersnieuwstraat 41, 9000 Gent, \\ Belgium \\ Correspondence should be addressed to Maria Lucia Scarpello,marialucia.scarpello@ugent.be
}

Received 20 September 2011; Revised 8 March 2012; Accepted 8 March 2012

Academic Editor: Carles Fernández-Prades

Copyright (C 2012 Maria Lucia Scarpello et al. This is an open access article distributed under the Creative Commons Attribution License, which permits unrestricted use, distribution, and reproduction in any medium, provided the original work is properly cited.

\begin{abstract}
A novel high-gain textile antenna array system, fully integrated into a rescue-worker's vest and operating in the Industrial, Scientific, and Medical wireless band $(2.4-2.4835 \mathrm{GHz})$, is presented. The system comprises an array consisting of four tiptruncated equilateral triangular microstrip patch antennas (ETMPAs), a power divider, line stretchers, and coaxial cables. The array is vertically positioned on the human torso to produce a narrow beam in elevation, as such reducing fading and allowing to steer the maximum gain in a small angular sector centered around the broadside direction. To allow simple low-cost beam steering, we specifically minimize mutual coupling by using a relative large distance between the patches and by selecting the ETMPA element as the most suited topology from three potential patch geometries. Moreover, we investigate the stability of return loss and mutual coupling characteristics under different relative humidity conditions, when bending the array, when placing the system on-body, and when covering it by different textile layers. Reflection coefficient and gain patterns are simulated and measured for the antenna system in free space and placed on the human body.
\end{abstract}

\section{Introduction}

Garments present a large platform for deploying smart textile systems combining textiles, electronics, and antennas. Being flexible, light weight, and conformable to the wearer, a communication system based on textile antennas can be fully integrated into clothing [1-5]. For example, one type of application for wearable antenna systems involves monitoring the activity and life signs of rescue workers active in the field, with the electronics being integrated into protective garments, setting up the off-body wireless communication link between the wearer and the closest base station [6]. In the literature, much research has been published about textile antennas based on a single radiating element, operating in the $2.45 \mathrm{GHz}$ Industrial, Scientific, and Medical (ISM) band (2.4-2.4835 GHz) [7-10], achieving good performance in terms of reflection coefficients and gain. The state of the art of research in wearable antenna arrays shows that a $3 \times 3$ fabric Electromagnetic Band Gap (EBG) antenna array with an overall thickness of $4.48 \mathrm{~mm}$ and size of
$120 \times 120 \mathrm{~mm}^{2}$ can cover the $2.45 \mathrm{GHz}$ ISM band, with a maximum measured gain of $6.4 \mathrm{dBi}$ at $2.45 \mathrm{GHz}$ in free space [11]. In [12] a four-element microstrip array on a $3 \mathrm{D}$ woven composite, operating at $1.54 \mathrm{GHz}$, is presented, but its gain is really low, and even not indicated. In [13], a twoelement microstrip parallel array on a 3D orthogonal woven composite is studied, operating at $1.5 \mathrm{GHz}$ with a gain equal to $6.4 \mathrm{dBi}$.

In [11-13], the mutual coupling between the adjacent elements of the array is not studied, whereas in this paper, we present the design and characterization of a 4-port textile antenna array system, able to operate in a wireless body and personal area network, in the $2.45 \mathrm{GHz}$ ISM band. For the first time in the literature, a complete study of the mutual coupling between the adjacent elements of the wearable array is performed when the array is in planar and bent state. The presented textile array is able to provide more reliable wireless off-body links, thanks to its array gain and its steerable narrow main beam. This is realized by deploying the array vertically on the human torso, allowing to steer 
the main beam in elevation within a narrow angular sector around the broadside direction, that is, away from the rescue worker and with the main beam pointing towards the closest base station, as requested by its intended application. The large aperture of the array leads to beam confinement, which in turn results in a smaller number of propagation paths and thereby less fading.

To achieve simple low-cost beam steering, we adopt a Uniform Linear Array (ULA) topology, composed of fourpatch antennas. Three different patch topologies are investigated in order to choose the one yielding the lowest mutual coupling between two adjacent elements. The simulated topologies are tip-truncated Equilateral Triangular Microstrip Patch Antenna (ETMPA), square ring patch antenna, and rhomboidal ring patch antenna. First, the distance between patches is chosen to be $3 / 4 \lambda$, where $\lambda$ is the freespace wavelength, to minimize mutual coupling between two adjacent elements, while allowing beamsteering within an angle of $20^{\circ}$ around broadside without grating lobes. Second, it is decided to adopt the ETMPA topology-for the first time in the literature chosen as radiating element for a textile antenna array system-since the ETMPA exhibits lower mutual coupling between adjacent elements than the other two patch topologies. Third, when deploying the system on-body, it is verified by real-life measurements that mutual coupling levels remain low under different adverse conditions: bending, varying relative humidity conditions, coverage by different textile layers, and proximity effects due to the body of the wearer.

Section 2 presents the design of the textile array. First, the choice of the array topology is explained, then a comparison between three different patch topologies is performed by means of full-wave simulations performed on the different array topologies. This comparison results in the optimal topology to be adopted as antenna element for the array in the remainder of the paper. Next, an accurate study of the textile array with the chosen patch topology is performed. In this section, it is also shown that the design choices lead to reliable off-body communication links, thanks to reduced fading. In Section 3, we show the simulations and measurements performed on the complete system, being a ULA connected by four coaxial cables to four line stretchersproviding the phase shifts necessary for beam steering - and a commercial four-way power divider. The influence of the relative humidity $(\mathrm{rH})$ on the reflection coefficient of the antenna array is discussed based on reliable and reproducible measurements in a controlled environment. Reflection coefficients and gain of the array are simulated by means of CST Microwave Studio and measured in free space and on the human body. Additional measurements of gain are carried out when the array is operating in other reproducible, but potentially adverse, conditions, demonstrating the array's functionality while being placed inside a firefighter jacket. For the evaluation of performance and safety issues, the 10$\mathrm{g}$ averaged Specific Absorption Rate (SAR) is calculated and compared to the ICNIRP [14] and FCC guidelines [15]. A comprehensive study of the mutual coupling between each patch of the antenna and the other three elements is carried out when the antenna is in planar and bent state, and

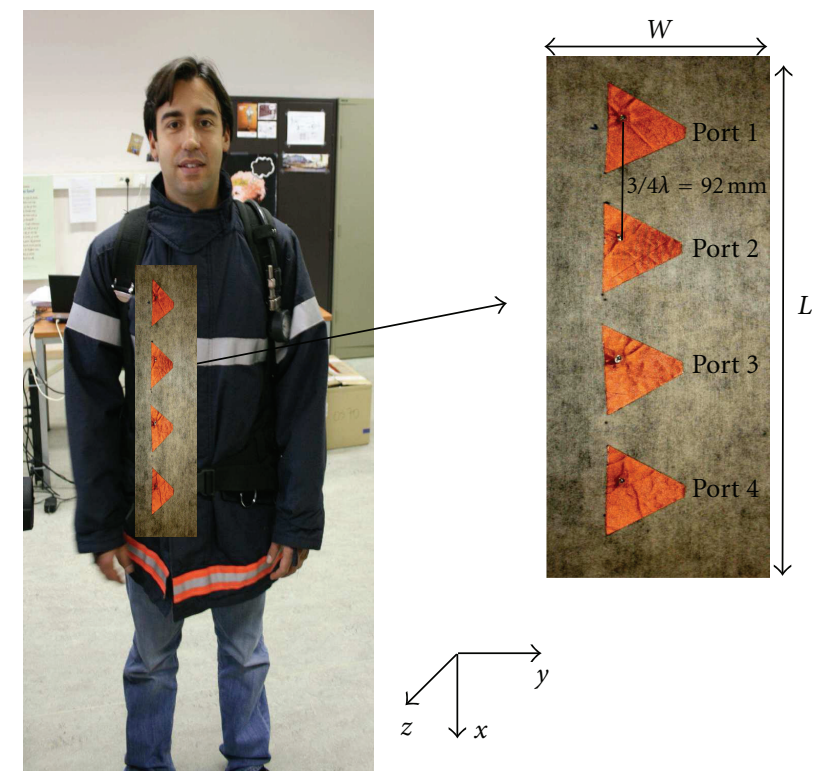

FIGURE 1: Top view of the textile antenna array and its position on the human body. (Note that in this figure, for illustrative purposes, the array is shown exposed on top of the garment. In reality, however, it is integrated into the garment.).

under different $\mathrm{rH}$ conditions. In Section 4, conclusions are summarized.

\section{Design of the Textile Antenna Array}

We now present a textile array providing a higher gain and offering more reliable communication links in a multipath environment than a single textile patch antenna. Therefore, we carefully chose the array and antenna element topology with the aim of maximizing the antenna aperture and minimizing the mutual coupling between two adjacent elements of the array.

2.1. Choice of Array Topology. We adopted a ULA topology, composed of four single patch antennas. The textile array is meant to be worn by a person, and it is vertically positioned on the human torso, as shown in Figure 1, in between two layers of garment, to avoid being directly in touch with the skin of the person. The array aperture was chosen in order to fully exploit the large platform presented by the garment. Deploying a vertical array allows to confine the energy within a narrow beam, centered around the azimuth plane. The vertical array offers limited steering capabilities of the beam maximum in a narrow angular sector, centered around the broadside direction. The total size of the array is $480 \times$ $180 \mathrm{~mm}^{2}$ and the distance between the four patches is $3 / 4 \lambda$, where $\lambda$ is the free-space wavelength, being approximately $122 \mathrm{~mm}$, as shown in Figure 1. The choice of $92 \mathrm{~mm}$ between two consecutive feeding points is a convenient choice as the complete array should be fit vertically on the human torso. Also the rather large spacing between two elements is chosen to minimize mutual coupling, while still allowing limited steering, and to yield a low-cost array with a large aperture. 


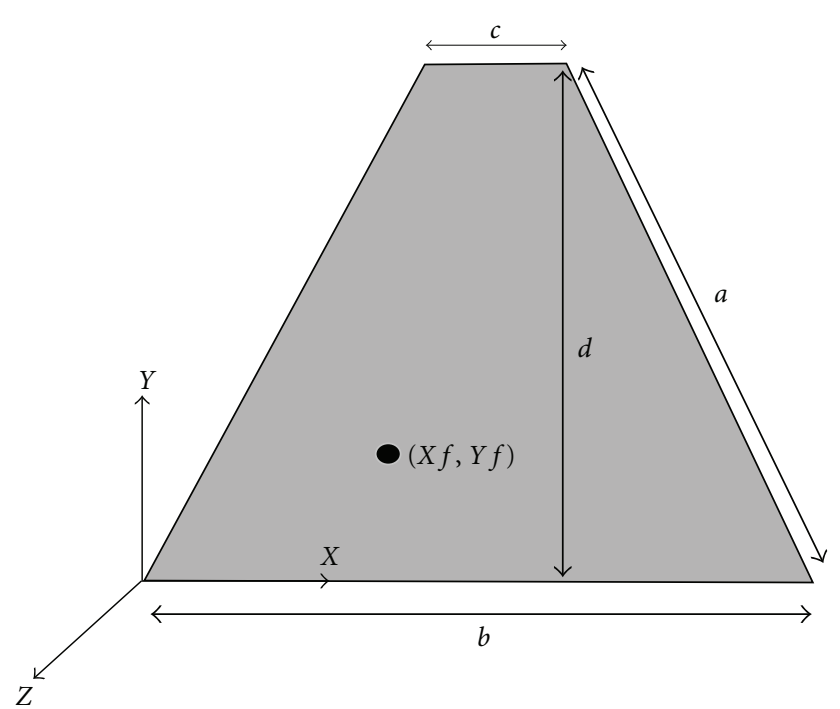

Figure 2: ETMPA.

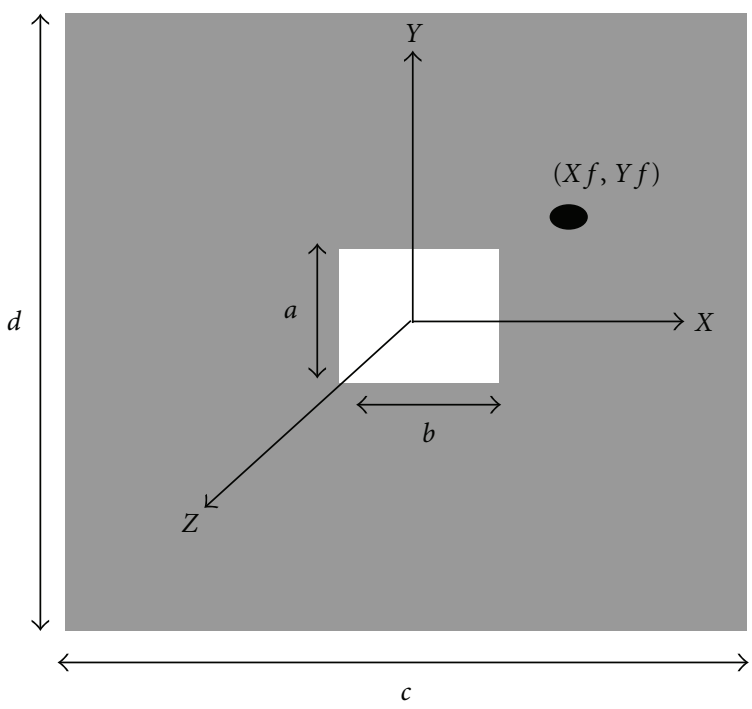

FIGURE 3: Square ring patch antenna.

2.2. Choice of Antenna Element Topology. In order to obtain the optimal performance from the chosen antenna array topology, we carefully investigated three antenna element topologies: tip-truncated ETMPA, square ring patch, and rhomboidal ring patch. Each single radiating element is shown in Figures 2, 3, and 4, and their dimensions are listed in Table 1. Four equal radiating elements are part of one ULA. Both the patch and ground plane of the array are made of Flectron, a breathable and highly conductive electrotextile material, being a copper-plated nylon fabric with a surface resistivity of less than $0.10 \Omega / s q$. The three ULAs are simulated on a polyurethane protective foam, called "Azzurri", manufactured by Brunet Lion [16], whose thickness is $3.55 \mathrm{~mm}$ and which is typically used in firefighters jackets. The dielectric characteristics of the substrate are $\epsilon_{r}=1.19$ and $\tan \delta=0.003$.

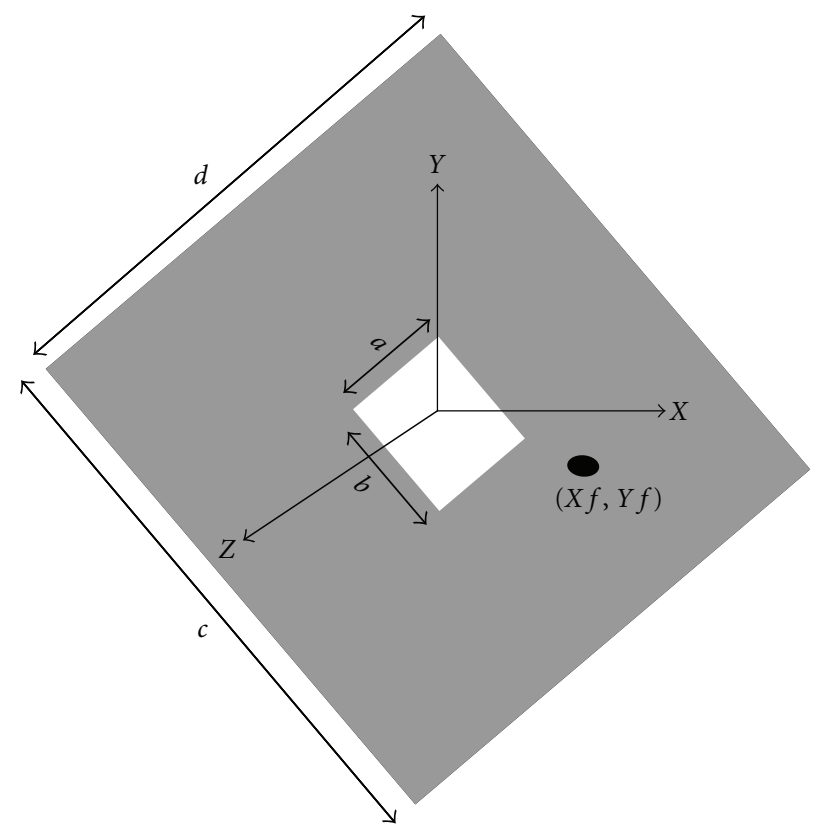

FiguRE 4: Rhomboidal ring patch antenna.

TABLe 1: Patch dimensions of the three antenna element topologies on Azzurri foam, used in the simulation study.

\begin{tabular}{lccc}
\hline & ETMPA $(\mathrm{mm})$ & Square $(\mathrm{mm})$ & Rhombus $(\mathrm{mm})$ \\
\hline$a$ & 60.1 & 8.4 & 8.4 \\
$b$ & 69 & 9.5 & 9.5 \\
$c$ & 8 & 51.52 & 51.93 \\
$d$ & 52.8 & 48.44 & 48.85 \\
$X f$ & 27.5 & 8.29 & 10.03 \\
$Y f$ & 10 & 5.56 & -2.24 \\
\hline
\end{tabular}

The substrate and patch dimensions were chosen to satisfy the following design criteria within the entire $2.45 \mathrm{GHz}$ ISM band:

$$
\begin{array}{r}
\left|S_{i j}\right|<-10 \mathrm{~dB} \text { for } i=j, \\
\left|S_{i j}\right|<-25 \mathrm{~dB} \text { for } i \neq j, \\
i, j=1,2,3,4,
\end{array}
$$

where $i$ and $j$ indicate the port numbers of the antenna array (Figure 1) and the $S_{i j}$ denote the pertinent S-parameters. In Figure 5, the simulated return loss of one patch of each one of the three ULAs is indicated and, in correspondence to (1), the ISM band is completely covered for the three topologies. Figure 6 shows the mutual coupling level between the first and the second antenna element obtained for each of the three arrays and it is seen that (2) is satisfied. The lowest mutual coupling value, however, is obtained for the ULA with the tip-truncated ETMPA elements. Using this topology, $\left|S_{12}\right|$ remains below $-30 \mathrm{~dB}$ within the entire ISM band. This margin of $5 \mathrm{~dB}$ with respect to constraint (2) is necessary as Figure 6 is the result of a simulation, and it can be expected 


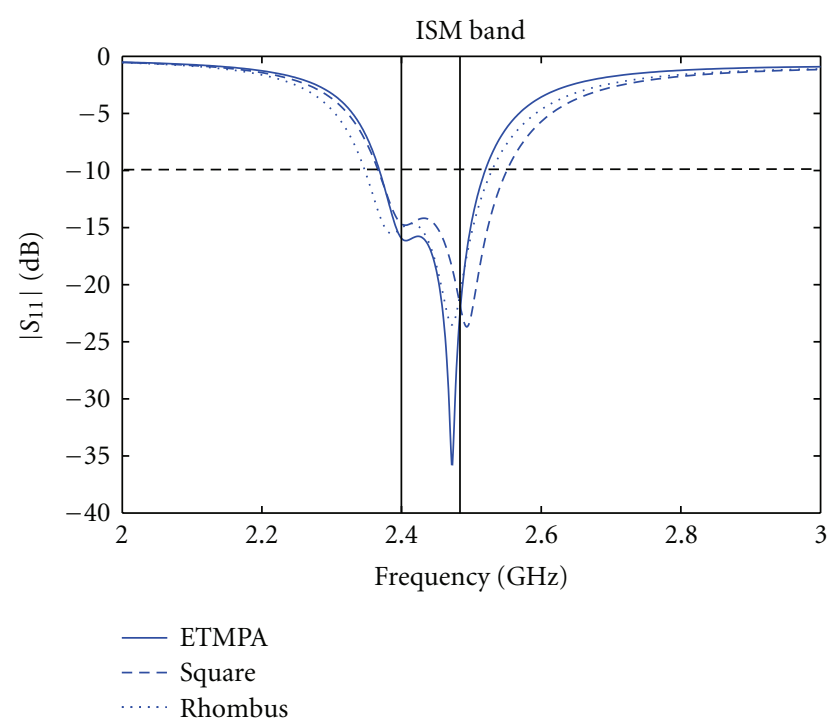

Figure 5: Simulated reflection coefficients of one port of the ULA array: tip-truncated ETMPA (full line), square ring patch (dashed line), and rhomboidal ring patch (dotted and dashed line).

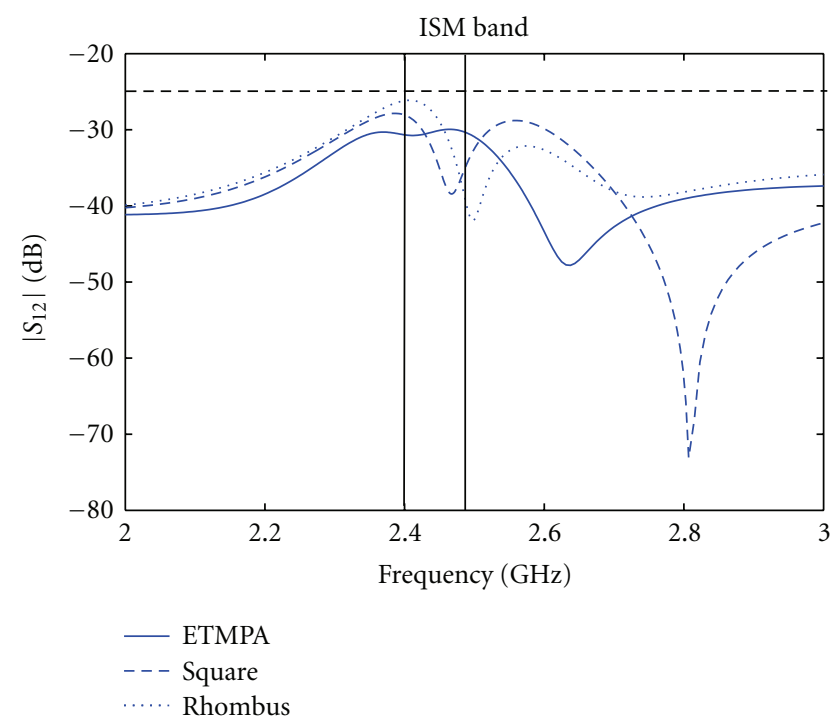

Figure 6: Simulated mutual coupling between the first and second port of the ULA array: tip-truncated ETMPA (full line), square ring patch (dashed line), and rhomboidal ring patch (dotted and dashed line).

that actual measured mutual coupling values of the manufactured array will be higher (see Section 3.4). Therefore, we proceed our study with ULAs consisting of tip-truncated ETMPA elements. The triangular patch, used as antenna element, not only guarantees low mutual coupling between adjacent elements, it also provides radiation characteristics similar to a rectangular microstrip antenna while occupying a smaller area. It is frequently used as a microstrip element, microstrip radiator or for array design on rigid substrates [17], but never before on a textile substrate. By cutting off
TABLe 2: Patch dimensions of the tip-truncated ETMPA on the Azzurri and cotton substrates, used as antenna elements in the array prototypes.

\begin{tabular}{lcc}
\hline & Azzurri $(\mathrm{mm})$ & Cotton $(\mathrm{mm})$ \\
\hline$a$ & 60.1 & 51.3 \\
$b$ & 69 & 53 \\
$c$ & 8 & 6.6 \\
$d$ & 52.8 & 45.8 \\
$X f$ & 27.5 & 20.8 \\
$Y f$ & 10 & 7.7 \\
\hline
\end{tabular}

a triangle from one of the tips of the patch $[18,19]$, the size of the ETMPA was further reduced.

2.3. Antenna Array Design and Prototyping. The complete array was hand cut and glued. The four tip-truncated ETMPAs are fed by means of $50 \Omega$ coaxial SMA connectors, which are manually soldered. To be able to study the stability of the array characteristics in different relative humidity conditions (Section 3), the optimal array topology is implemented on two different nonconductive textile substrates, having very different Moisture Regains (MR), that is, the above introduced polyurethane protective foam, "Azzurri," and a cotton fabric. The characteristics of the Azzurri foam are of course the ones presented in Section 2.2. The cotton substrate, composed of four thin layers of cotton fabric to provide a large enough bandwidth, has a total thickness of $2.27 \mathrm{~mm}$ and its dielectric characteristics are $\epsilon_{r}=1.94$ and $\tan \delta=0.02$. Geometrical parameters of the patches are listed in Table 2 and indicated in Figure 2. Obviously, also for the implementation on cotton, the patch dimensions and choice of substrate material and thickness are chosen to satisfy (1) and (2). When deployed, both textile array implementations - on cotton and on Azzurri-result to be light weight, conformal to the body, and easy to bend.

2.4. Benefit of the Array in a Multipath Environment. It is now demonstrated that the above design choices indeed lead to more reliable off-body communication links. In a line-of-sight scenario in absence of multipath, the use of a vertical array is expected to provide $6 \mathrm{~dB}$ of array gain compared to a single textile patch, thanks to the confinement of the beam in elevation. In the receive case, an extra $6 \mathrm{~dB}$ of gain is obtained as the signal is received by the four antenna elements of the array. When deploying the array in a multipath environment, the beam confinement along the elevation direction will reduce the number of paths contributing to fading, and it is expected that fluctuations due to fading will be smaller compared to the single textile antenna case. This statement was validated by means of the ray tracer Winprop V7.40 of AWE Communications. We considered a wireless link in an office building of Ghent University, of which the floor plan is shown in Figure 7. A $2.45 \mathrm{GHz}$ ISM band access point is located at one end of the corridor, being position $\mathrm{C}$ in Figure 7. A firefighter equipped with a textile antenna system as shown in Figure 1 walks 


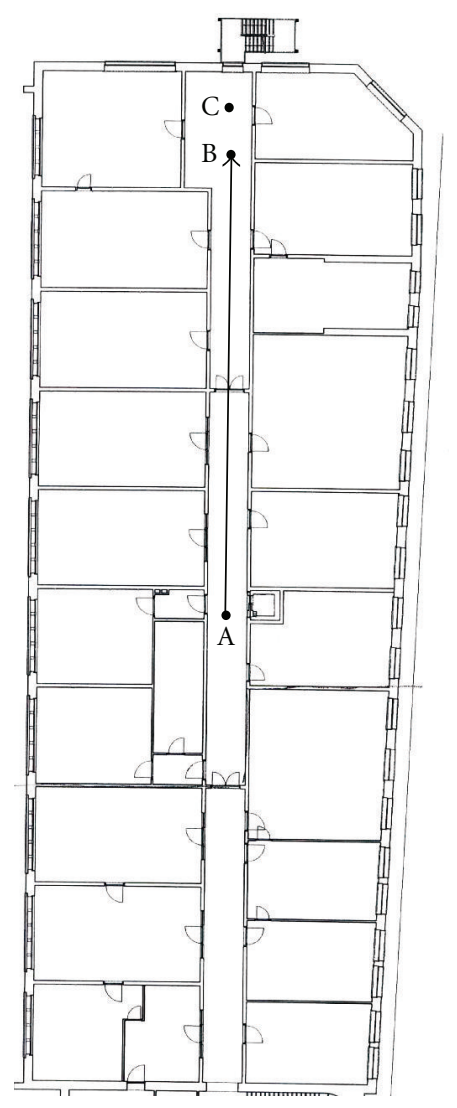

FIGURE 7: Plan of the indoor environment: the person wearing the firefighter jacket walks for $21 \mathrm{~m}$ from the position A to B, while the transmitting access point antenna is positioned in location $\mathrm{C}$.

towards the access point from position A to position B, for a total distance of $21 \mathrm{~m}$. Figure 8 displays the amplitudes of the signal received by a single textile patch and by the vertical textile array after beamforming, taking into account the antenna's gain patterns shown in Figures 12 and 13. This clearly demonstrates the benefits of beam confinement in elevation by the textile array. First, for the receive case, we clearly observe an array gain thanks to beamforming (Note that, for the transmit case, the full line will decrease by $6 \mathrm{~dB}$, since the available power must be equally distributed over the four antenna elements). Second, the large fading dips seen in the dotted curve for the single textile antenna are clearly reduced by the narrow beam generated by the vertical array (full curve). Hence, both in outdoor and indoor scenarios, the proposed system will provide more reliable links than a single textile patch.

\section{Simulation and Measurements}

To feed the array in the system, a commercially available conventional four-way power divider is used, attached to the array by means of four thin flexible coaxial cables containing four line stretchers, as shown in Figure 9, to guarantee that (i) the same power is injected at each port of the array and (ii) beamsteering is implemented by manipulating the phases

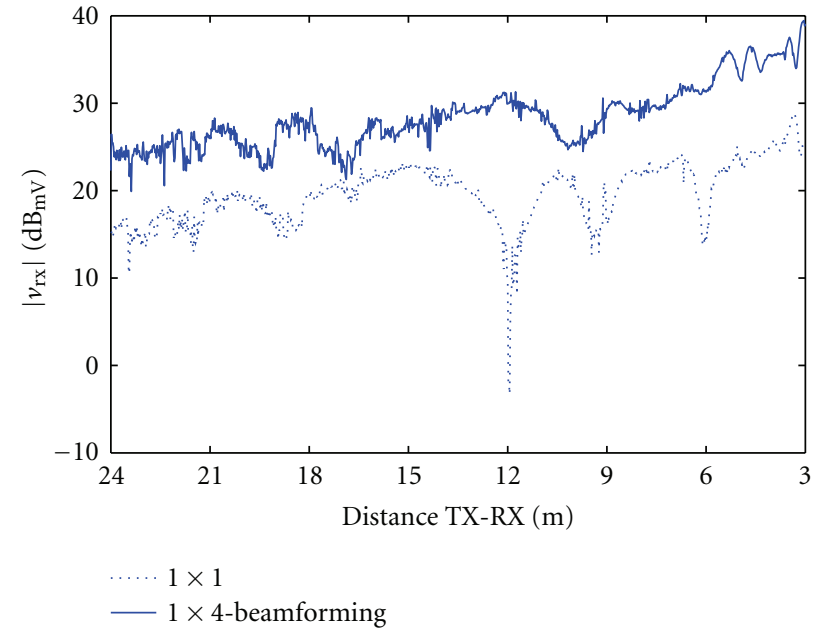

FIGURE 8: Amplitude of the signal received by the array after beamforming (full line) versus the amplitude of the signal received by a single patch antenna (dotted line), as a function of distance to the transmit antenna.

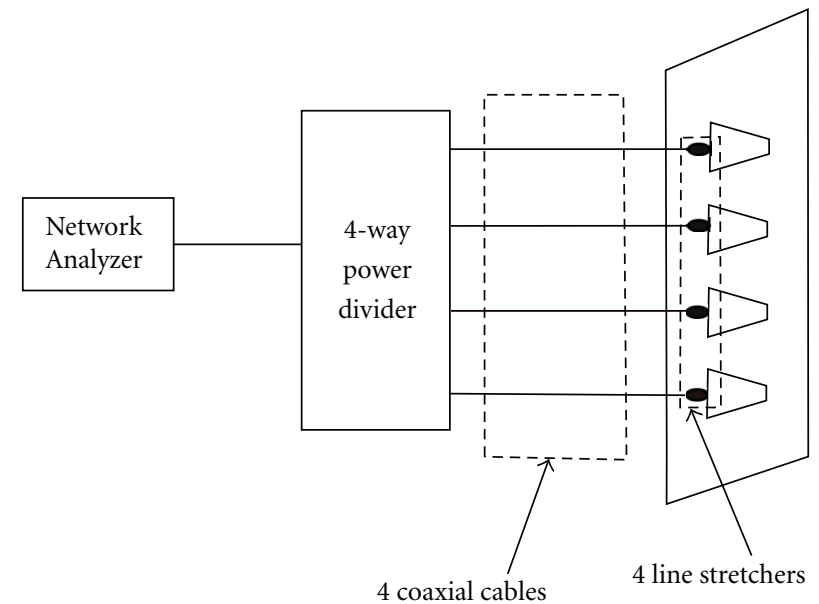

Figure 9: Entire antenna system, consisting of a four-way power divider, four cables, four line stretchers, and the textile antenna array.

of the line stretchers. We now evaluate the performance of the complete textile array system by means of simulations and measurements. The simulations are all performed using the 3D simulator CST Microwave Studio. First, the reflection coefficient of the array system is measured for varying $\mathrm{rH}$ conditions created in a controlled environment. Second, the array is placed in the anechoic chamber for the measurement of gain in free space and when being deployed on the human torso. Third, a comprehensive mutual coupling study is carried out when the antenna is in planar and bent state and while varying the $\mathrm{rH}$ conditions.

3.1. Reflection Coefficient Measurement under Varying Relative Humidity Conditions. Environmental changes, such as humidity, can influence the performance of textile antennas. 


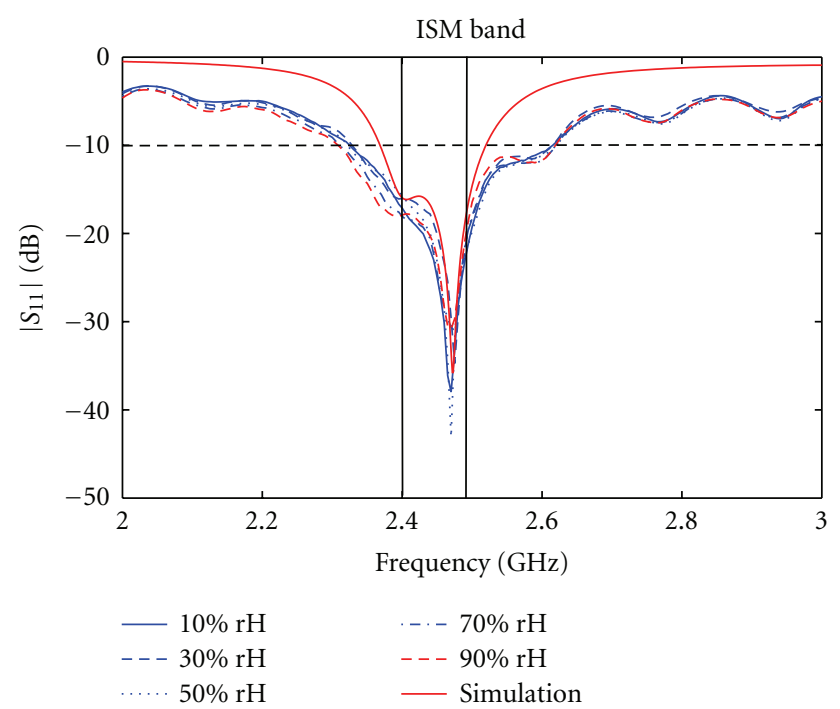

Figure 10: Array on Azzurri: reflection coefficients, simulation (full line) versus measurements (dashed line).

For example, as fire fighters are subjected to heat and heavy physical exertion, there is an increase of thermal sweating, substantially changing the $\mathrm{rH}$. Therefore, in this paper, the influence of $\mathrm{rH}$ on the performance of the array system is studied. The choice of Azzurri foam and cotton fabric, as nonconductive antenna substrates, is made as these are textile materials characterized by a small and a large moisture regain (MR), respectively: MR of Azzurri is $1.7 \%$, whereas the MR of cotton is $6.9 \%$. To obtain reproducible and reliable measurements, the two implementations of the array were conditioned for 24 hours in a climate test cabinet (WK 350 from Weiss Technik) at a temperature of $23^{\circ} \mathrm{C}$ and the $\mathrm{rH}$ was varied each day. At the end of each day, they were taken out of the test cabinet, one by one, and immediately measured with an Agilent RF Network Analyzer placed in the nonanechoic lab environment where the climate test cabinet is located to prevent any possible change in the conditions of the antenna. Note that, to cover the complete ISM band, the required $-10 \mathrm{~dB}$ impedance bandwidth of the antenna is 83.5 MHz in the [2.4-2.4835] GHz ISM band. The reflection coefficient of the entire antenna system, consisting of the four-way power divider, the four line stretchers, and the four coaxial cables (Figure 9), attached to the array implemented on either of the two substrates, were measured for an $\mathrm{rH}$ equal to $10 \%, 30 \%, 50 \%, 70 \%$, and $90 \%$. Figures 10 and 11 show the measured reflection coefficient of the array on Azzurri and on cotton, respectively, in comparison with the simulated reflection coefficient. The difference between simulated and measured results in Figures 10 and 11 stems from the losses that are present in the complete system. The insertion loss (IL) of the power divider is approximately $6 \mathrm{~dB}$, the IL of each cable is $0.76 \mathrm{~dB}$ and the IL of each line stretcher is $0.2 \mathrm{~dB}$. In the simulations, the IL of the cables and line stretchers were not considered. On the one hand, the antenna on Azzurri exhibits a very stable performance, fully covering the ISM band. On the other hand, for the

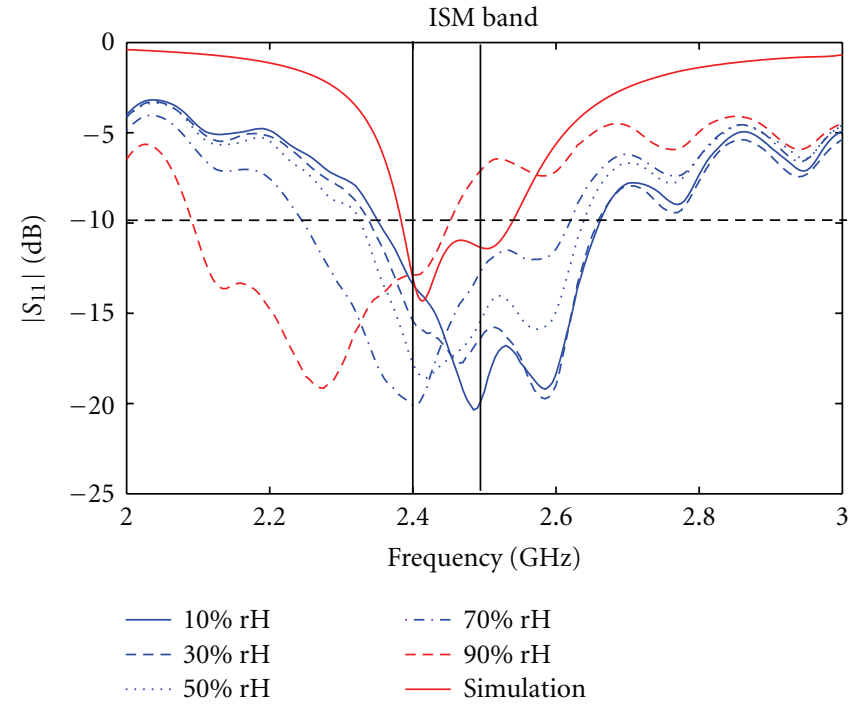

FIgURE 11: Array on cotton: reflection coefficients, simulation (full line) versus measurements (dashed line).

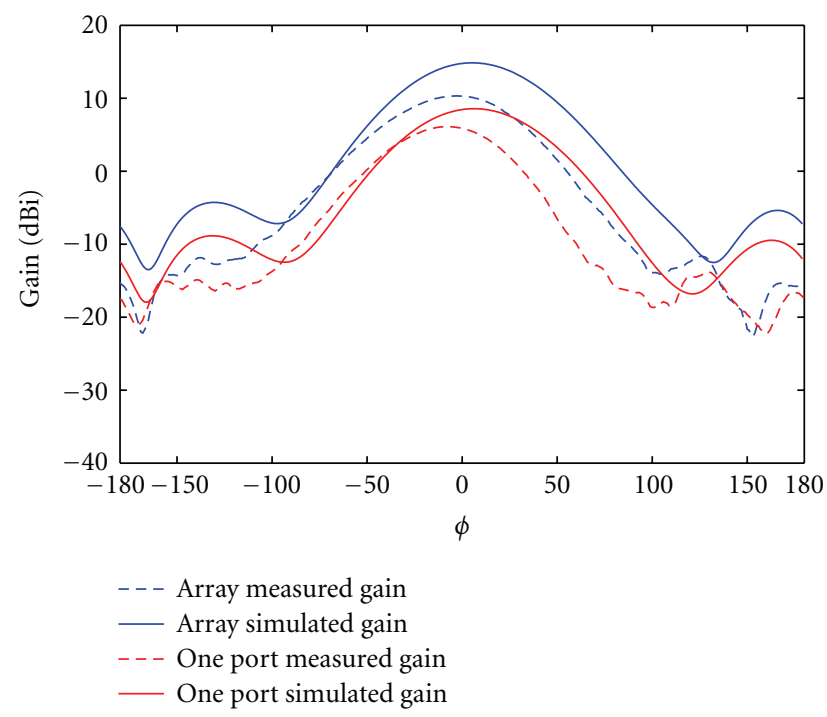

Figure 12: Array on Azzurri: gain pattern at $2.45 \mathrm{GHz}$, in the azimuth plane ( $y z$-plane), simulation versus measurement of the entire array and of one single patch.

antenna on cotton, the resonance frequency decreases with increasing $\mathrm{rH}$, and the ISM band is no longer covered when the $\mathrm{rH}$ is $90 \%$. This is related to the MR value of the two nonconductive substrates: in fact, when choosing a textile material as a substrate for a textile antenna suitable for integration into garment, the MR value of the textile must be taken into account, as indicated in [20]. When varying the $\mathrm{rH}$, the antenna efficiency of the array fabricated on Azzurri substrate remains nearly constant, whereas for the antenna elements of the array produced on the cotton substrate, the radiation efficiency decreases with increasing $\mathrm{rH}$ [21]. Predicting the change of the dielectric constant of substrates, due to changes in $\mathrm{rH}$, and predicting the 


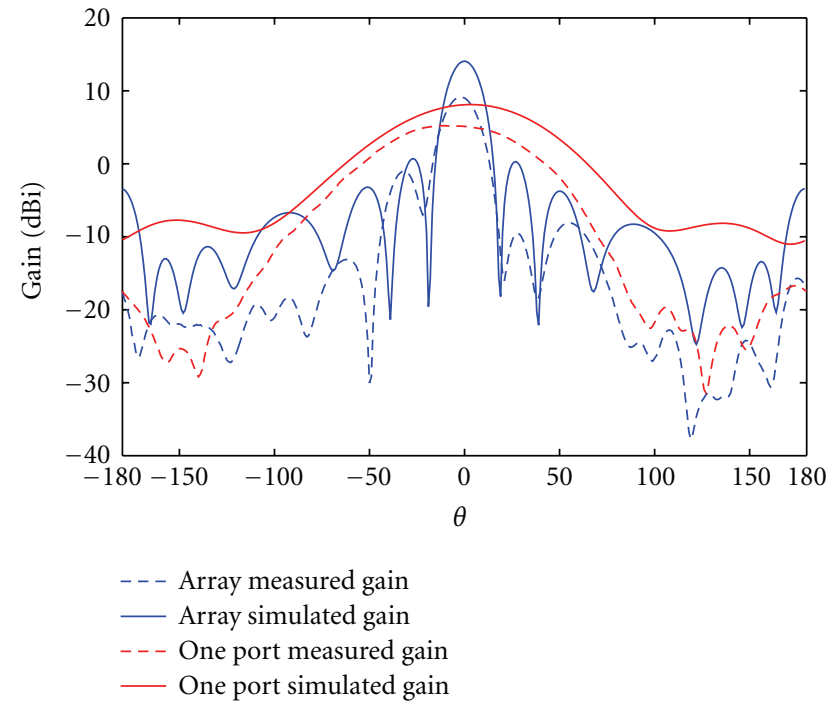

Figure 13: Array on Azzurri: gain pattern at $2.45 \mathrm{GHz}$, in the elevation plane ( $x z$-plane), simulation versus measurement of the entire array and of one single patch.

variation of the thickness of the substrates, due to swelling of, for example, the cotton fibers, would be possible by using techniques described in [22]. However, in this paper, we opted to skip this step and to immediately implement two designs, validating directly the end results by means of reliable measurements in a controlled environment. Given the lack of stability in the reflection coefficient of the array on the cotton substrate under varying humidity conditions, we decided to continue the study for the textile array implemented on Azzurri foam only.

3.2. Free-Space Measurements. To check if the present array on Azzurri is a better solution in terms of gain with respect to the single patch antenna, we performed measurements and simulations comparing the configuration where the four ports are equally fed by the four-way power divider to the one where only one of the four patches is excited, while the other three patches are connected to a $50 \Omega$ load. Figure 12 shows the gain pattern of the array at $2.45 \mathrm{GHz}$, in the azimuth plane ( $y z$-plane). The array's simulated gain is $6 \mathrm{~dB}$ larger than the simulated gain of the single patch antenna. This is a predictable result because the array is a four-port array, gaining $6 \mathrm{~dB}$ with respect to the single patch. In reality, this value cannot be achieved. The entire system, as illustrated in Figure 9, encompasses different elements, such as line stretchers, cables, and a power divider, which generate losses that are hard to avoid. Consequently, the measured gain is $4 \mathrm{~dB}$ larger than the gain of a single patch antenna. The simulated gain pattern has the beam steered along $\phi_{m}=0^{\circ}$, with $\phi_{m}$ indicating the angle $\phi$ in the $y z$-plane where the gain is maximum. The measured gain pattern exhibits a beam steered along $\phi_{m}=-4^{\circ}$. This deviation is due to the difficulty to position the entire antenna system (Figure 9) in the anechoic chamber for measurements. Nonetheless, this small positioning error does not result in major degradation of the

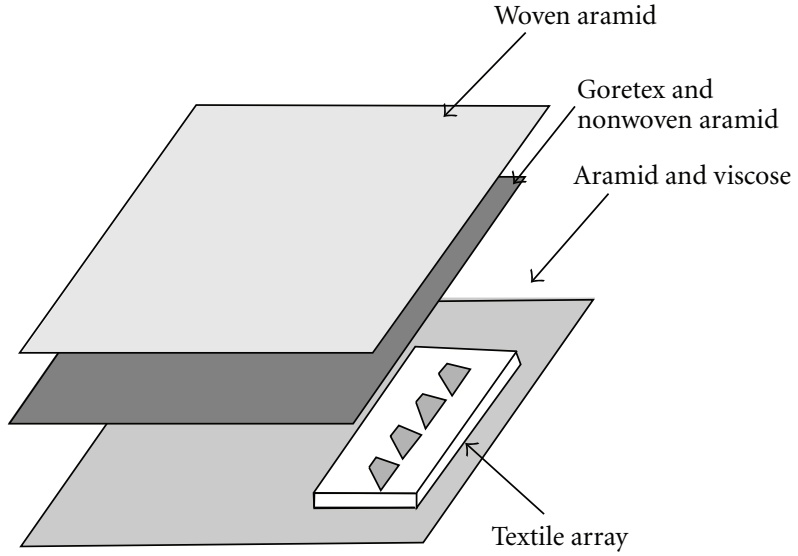

Figure 14: Antenna between textile layers composing the firefighter suit.

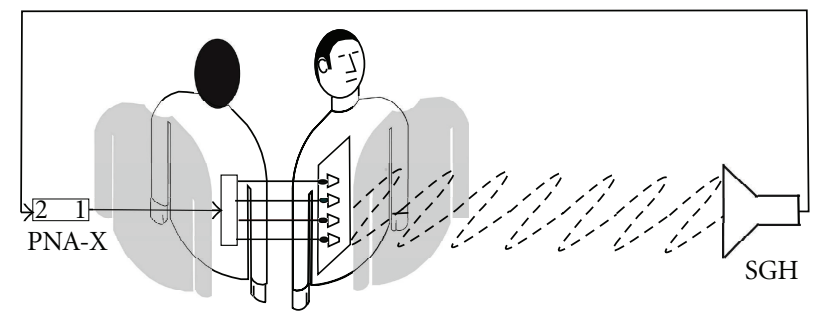

FIGURE 15: Entire antenna system placed on the human body: port 2 of the Agilent N5242A PNA-X Network Analyzer is connected to the Standard Gain Horn (SGH) and port 1 is connected to the 4port power divider.

array performance, given the sufficiently large beamwidth of the array. Figure 13 shows the gain pattern of the array at $2.45 \mathrm{GHz}$ in the elevation plane (xz-plane). The array's measured $3 \mathrm{~dB}$ beam width is equal to $18^{\circ}$ and similar conclusions concerning the difference between measured and simulated gain, as for Figure 12, can be drawn.

3.3. On-Body Measurements. In real-life applications, the antenna is positioned vertically on the torso of the human body, to steer the beam poviding the maximum gain in a narrow angular sector in the broadside direction, where the base station is located. The array may be deployed in a firefighter jacket, whose composing textile layers are shown in Figure 14. The first layer on top of the array is a combined moisture and thermal barrier, made of Goretex and nonwoven aramid, and above it there is an outer shell fabric layer made out of woven aramid. Below the ground plane there is a third layer, composing the jacket liner, made out of aramid and viscose. The resulting effect on the gain was investigated by means of simulations, with the $3 \mathrm{D}$ simulator CST Microwave Studio, and by measurements.

During measurements, the layout shown in Figure 15 is adopted to place the entire antenna system on the human body: the array is integrated into the firefighter jacket, in front of the human torso, between the textile layers combined the suit. Four line stretchers are placed between the four SMA 


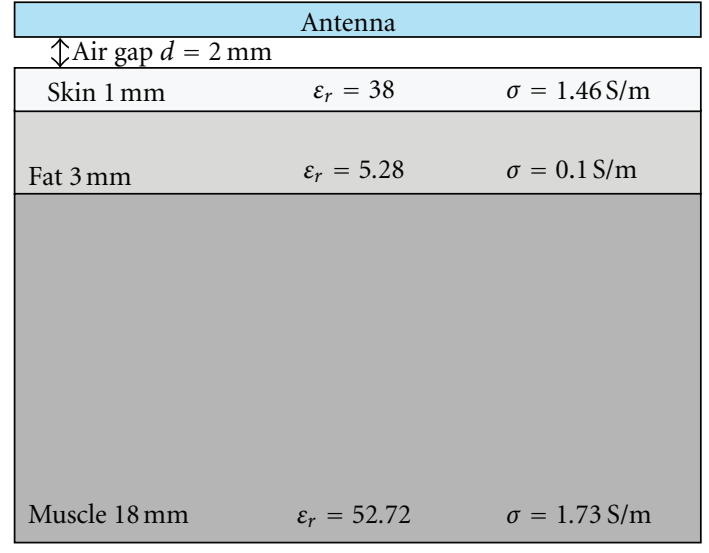

FIgURE 16: The textile array is placed on top of the model of a human body used for the simulations [23]. The dielectric values of three different human tissues, at $2.45 \mathrm{GHz}$, are those reported in [24-26].

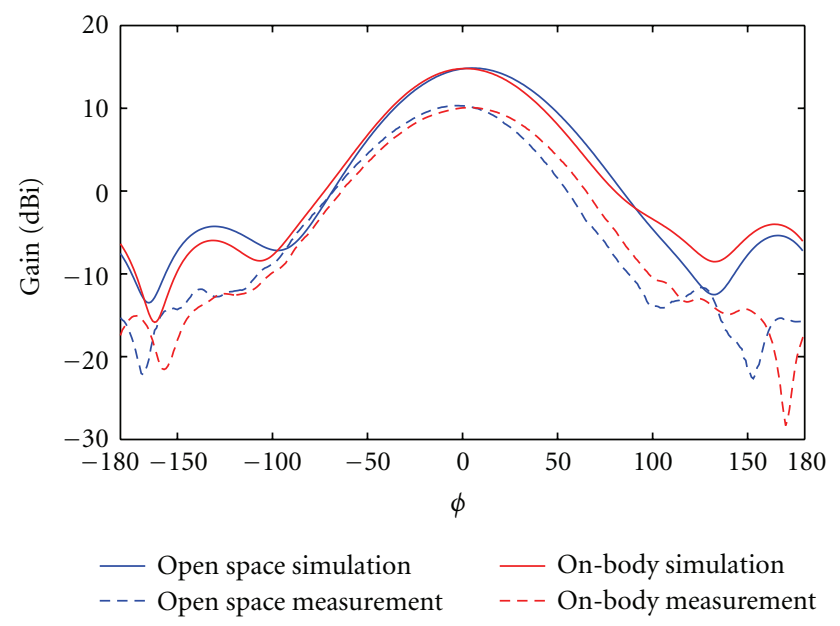

FIGURE 17: Gain pattern in the azimuth plane ( $y z$-plane) at $2.45 \mathrm{GHz}$ in open space and when the antenna is placed on-body in a firefighter jacket: simulations (full line) versus measurements (dashed line).

connectors and the four coaxial cables that are routed along the side of the chest to reach the back of the shoulder where the four-way power divider is located.

As for simulations, we rely on the model [23] depicted in Figure 16 to simulate the on-body situation: we use a thickness of $1 \mathrm{~mm}$ for the skin layer, $3 \mathrm{~mm}$ for the fat layer, and $18 \mathrm{~mm}$ for the muscle layer, together with the dielectric values of the three human tissues specified in Figure 16.

Figure 17 shows the simulated and measured gain patterns of the array in the azimuth plane ( $y z$-plane) when located in free space and when deployed on the human body. The two situations present similar gain patterns, indicating that the presence of the body does not degrade the performance of the array. For the on-body case, we obtain a maximum simulated gain of $14.7 \mathrm{dBi}$ versus $10.2 \mathrm{dBi}$ of maximum measured gain. This should be compared to $14.8 \mathrm{dBi}$ of simulated gain versus $10.3 \mathrm{dBi}$ of measured gain

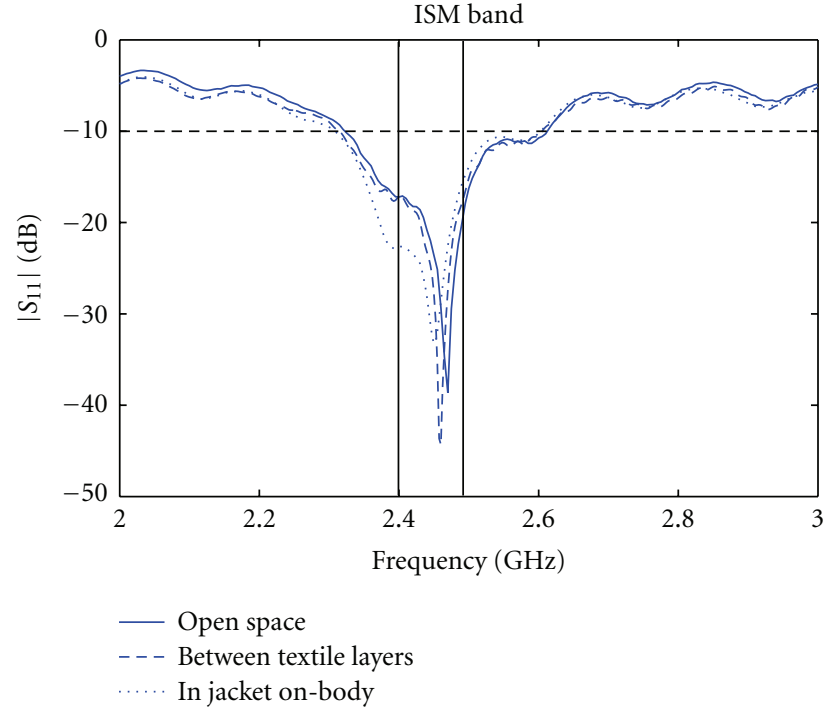

Figure 18: Measured reflection coefficients in three different situations: array in open space (full line), array placed between textile layers (dashed line), and array placed on-body in a firefighter jacket (dotted line).

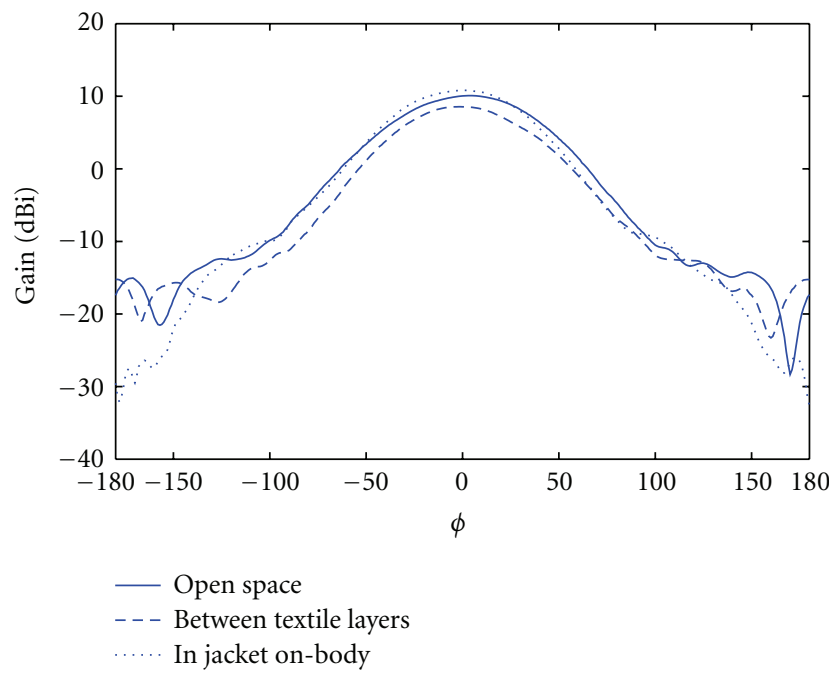

FIGURE 19: Measured gain pattern in the azimuth plane ( $y z$-plane) at $2.45 \mathrm{GHz}$ in three different situations: array in open space (full line), array placed between textile layers (dashed line), and array placed on-body in a firefighter jacket (dotted line).

in the free-space case. Note that, although here the pattern is steered along broadside, in the actual application, the steering capabilities of the array allow to adjust the direction of the main lobe.

Due to mechanical limitations of the measurement system in the anechoic chamber, on-body gain patterns in the elevation plane cannot be measured. Figures 18 and 19 show the measured reflection coefficient and the gain pattern in the azimuth plane ( $y z$-plane), respectively, of the textile array in three situations: open space, placed between the textile layers shown in Figure 14, and integrated into the firefighter jacket 


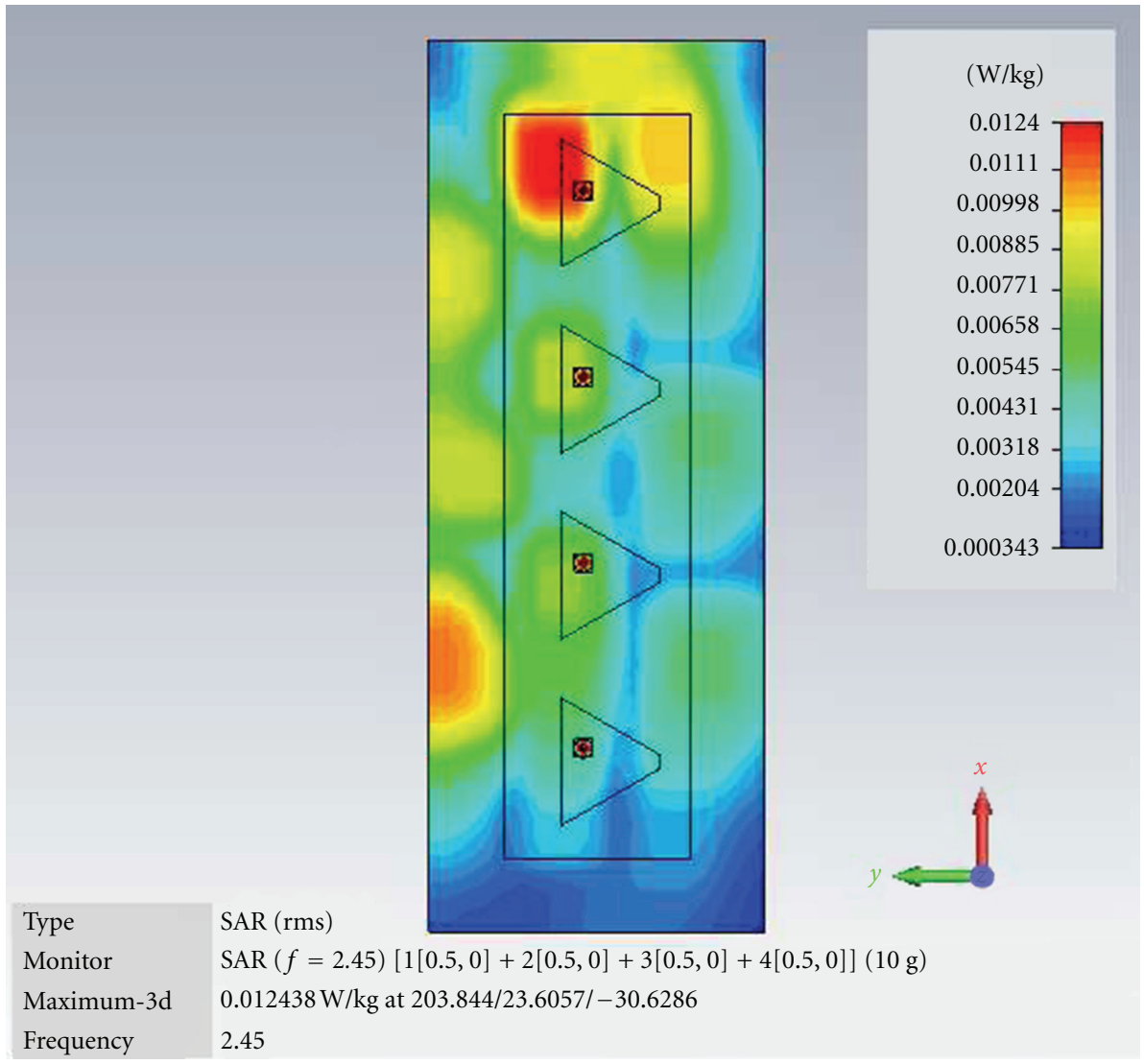

Figure 20: SAR distribution.

worn by a person. In all the three cases, the ISM band is fully covered and the gain patterns exhibit the same trend.

The human body has a very complex structure in terms of electrical parameters of each tissue, thus the energy of high frequency electromagnetic waves is absorbed by the human body with various intensity, depending on the part of the body and on the wave frequency. To analyze this problem and to guarantee that the antenna in this paper does not harm the person wearing it, the Specific Absorption Rate (SAR) is calculated, using the 3D simulator CST Microwave Studio, which computes the amount of energy absorbed by the human body. The human body model used for the simulations is shown in Figure 16. Figure 20 presents the SAR distribution in the $x y$-plane of the array at $z=0$ when the input power at each port is $0.5 \mathrm{~W}$. The 10 -g averaged SAR peak value is $0.0124 \mathrm{~W} / \mathrm{Kg}$. This value fulfills the ICNIRP [14] and FCC [15] guidelines for general public exposure.

3.4. Mutual Coupling Measurements. To assess the sensitivity of the mutual coupling in the array w.r.t. environmental conditions, more specifically, w.r.t. to changes in the relative humidity, two types of measurements are performed in a real nonanechoic lab environment after $24 \mathrm{~h}$ of conditioning in the climate test cabinet and the results are verified against the conditions (1) and (2): first, the reflection coefficient of each individual array element is measured, while terminating the other three elements with a $50 \Omega$ load; second, the mutual coupling between each pair of antenna elements is measured, while terminating the other two elements with a $50 \Omega$ load. Figure 21(a) shows the measured reflection coefficient at port 1 of the array, and Figures 21(b), 21(c), and 21(d) show the mutual coupling between port 1 of the antenna array and the other three ports, setting $\mathrm{rH}$ to be $20 \%, 40 \%, 60 \%$, and $80 \%$. It is observed that the array is rather insensitive w.r.t. to environmental conditions, always satisfying (1). For the other antenna elements (ports 2-4) a similar behavior is observed. Thanks to the careful design, the mutual coupling between the individual radiators remains low enough and (2) is always satisfied. Similar characteristics are obtained for the other combinations of port pairs, being 2 and 3;2 and 4; 3 and 4.

Next, the mutual coupling between each pair of ports of the array was measured when the array-in flat conditionis positioned in our anechoic chamber in three conditions, being in open space, between the textile layers composing the firefighter suit shown in Figure 14, and when it is fully integrated inside the firefighter jacket. Results are shown in Figures 22(a), 22(c), and 22(e). We note that, given the fact that the measurements are now performed in anechoic conditions, the mutual coupling levels are lower than the ones found in Figures 21(b), 21(c), 21(d) and that they vary little for the different setups. To verify the effects of bending on the mutual coupling between each pair of antenna elements, the array is bent around a plastic cylinder 


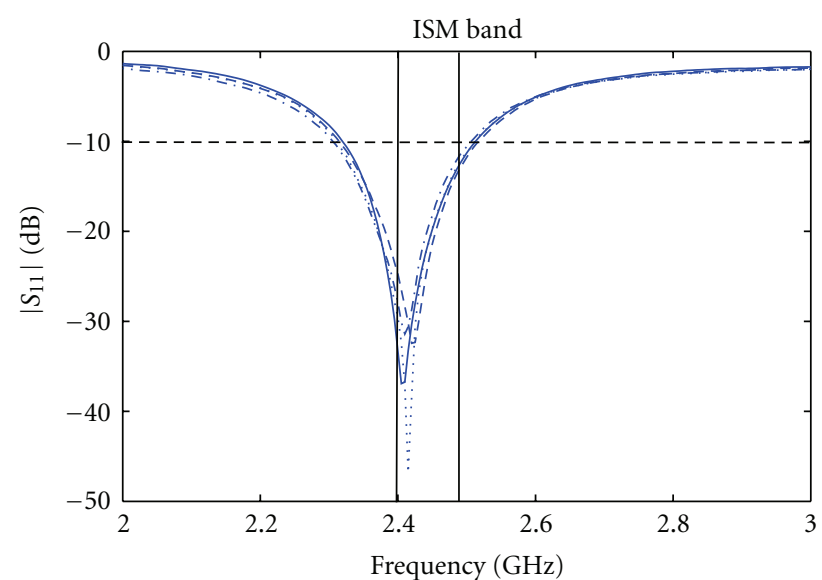

(a) Reflection coefficients at port 1

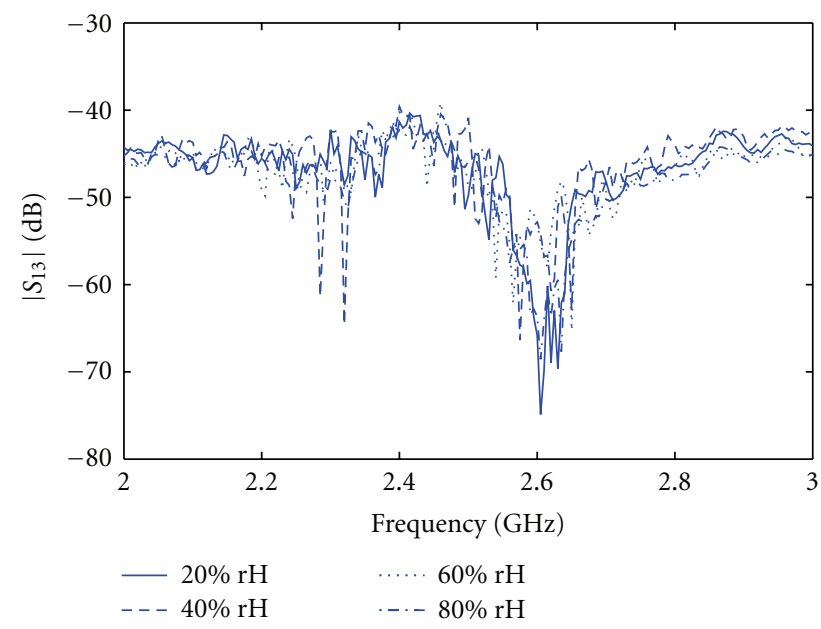

(c) Mutual coupling between port 1 and 3

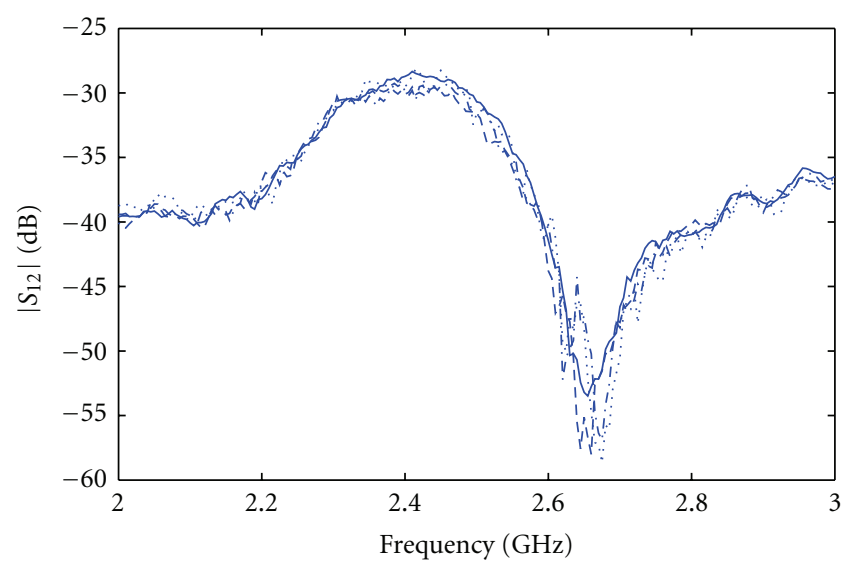

(b) Mutual coupling between port 1 and 2

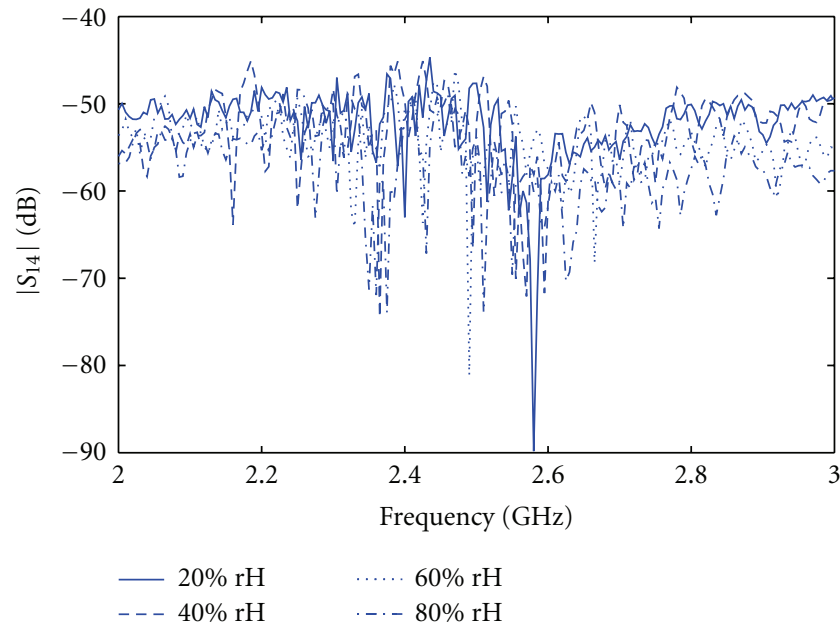

(d) Mutual coupling between port 1 and 4

FIGURE 21: S-parameter measurements after acclimatization of the array, measured in a nonanechoic environment: reflection coefficients at port 1 of the array and mutual coupling between port 1 of the array and the other three ports.

with a diameter of $15 \mathrm{~cm}$, oriented vertically, that is, along the $y$-axis, representing an extreme case where the array is bent around an arm. The mutual coupling was measured when the array is in open space and between the textile layers, composing the firefighter suit, shown in Figure 14. It is found that mutual coupling is not significantly affected by bending the textile array. Finally, we observe that both in planar and bent state, (2) is always satisfied. Similar characteristics are obtained for the other combinations of port pairs, that is, 2 and $3 ; 2$ and $4 ; 3$ and 4 .

\section{Conclusion}

The design, manufacturing, and measurements were discussed of a wearable system composed of a four-way power divider and line stretchers interconnecting a four-element ULA textile array consisting of tip-truncated ETMPAs implemented on two different nonconductive textile substrates, that is, cotton fabric and Azzurri foam, operating in the $2.45 \mathrm{GHz}$ ISM band. Thanks to the exclusive use of isolating and conductive textiles, the array is breathable, flexible and may be fully integrated into garments, worn by rescue workers or fire fighters, active in a disaster area. The array should be placed vertically on the human torso, to steer the beam providing the maximum gain in elevation angle within a narrow angular sector around the broadside direction, ensuring that the main beam points towards the base station. Thereto, a four-patch ULA was chosen with a relatively large spacing between the individual elements. This leads to a lowcost design with a large antenna aperture and low mutual coupling between the patches, allowing easy steering. The narrow antenna beam also results in less fading and, hence, reliable communication. Under reproducible $\mathrm{rH}$ conditions, realized in the climate test cabinet, measurements of the reflection coefficient of the array system were performed: the implementation of the array on Azzurri foam exhibits perfectly stable characteristics. For the implementation on cotton, however, the resonance frequency decreases with increasing $\mathrm{rH}$, and the ISM band is no longer covered when the $\mathrm{rH}$ is $90 \%$. Hence, when choosing a textile material as 


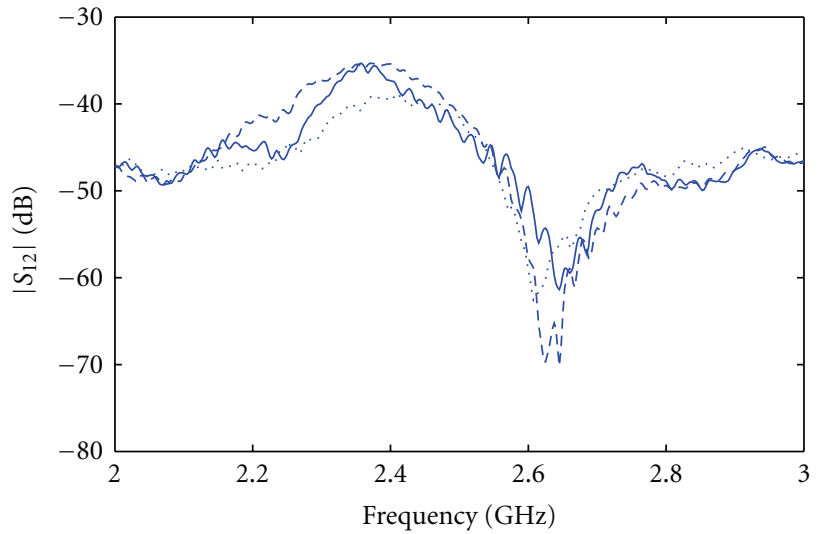

(a) Planar: mutual coupling between port 1 and 2

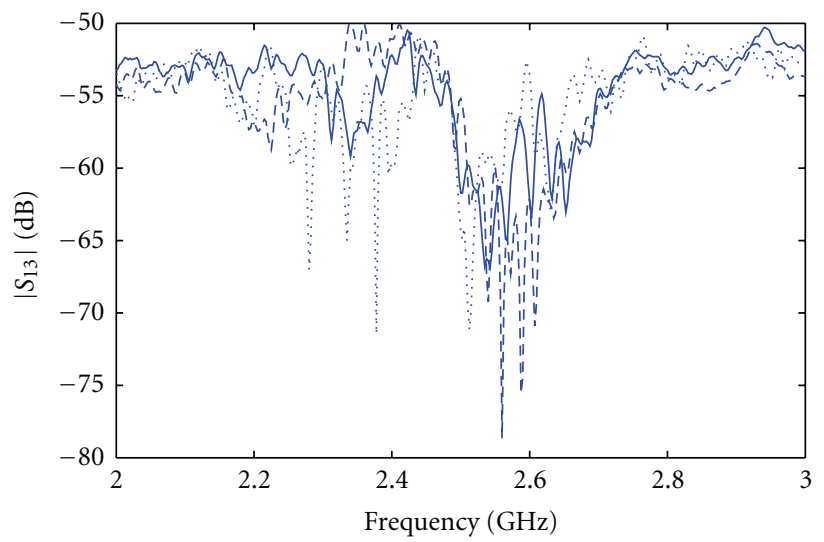

(c) Planar: mutual coupling between port 1 and 3

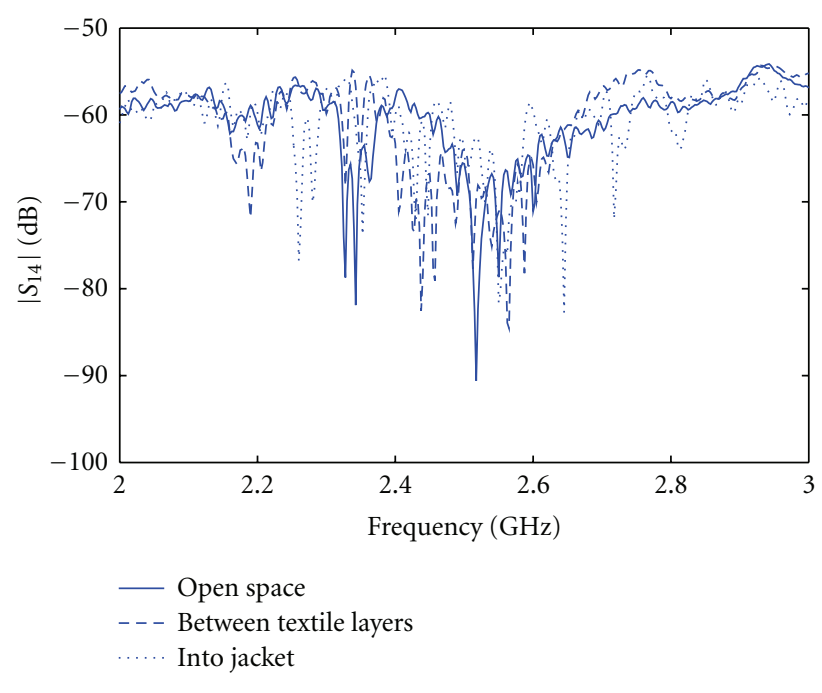

(e) Planar: mutual coupling between port 1 and 4

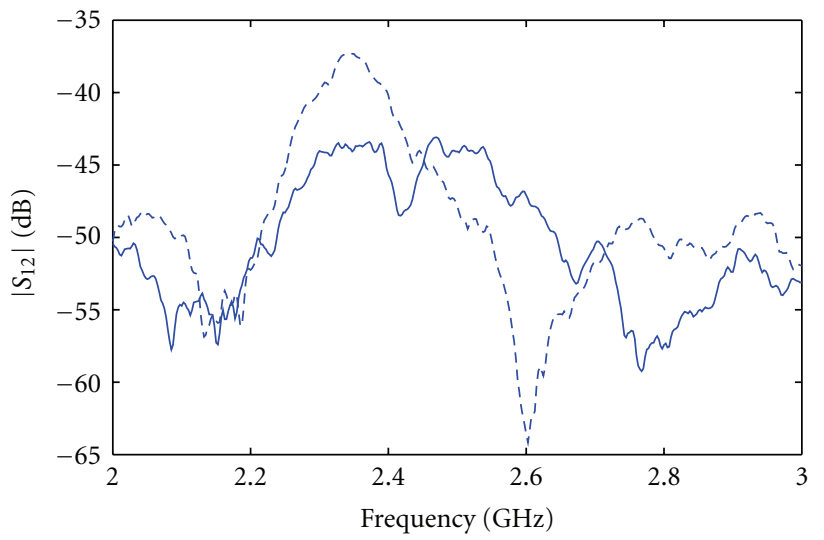

(b) Bent: mutual coupling between port 1 and 2

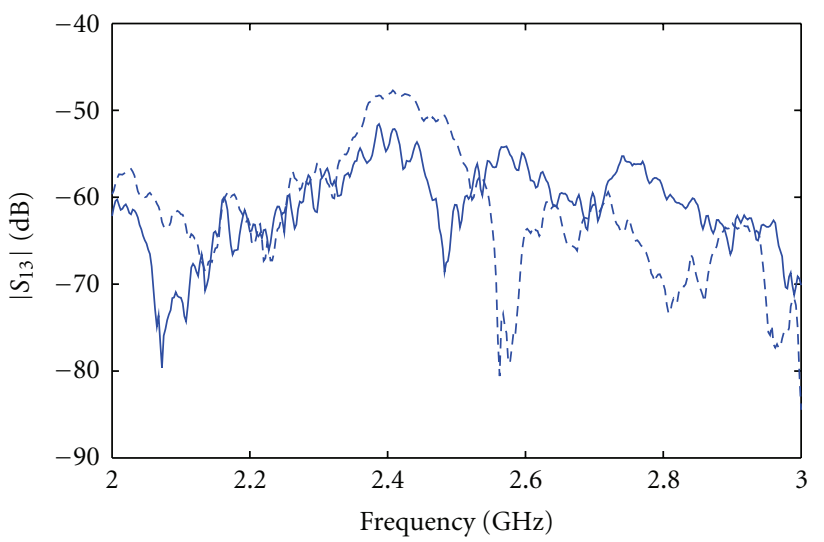

(d) Bent: mutual coupling between port 1 and 3

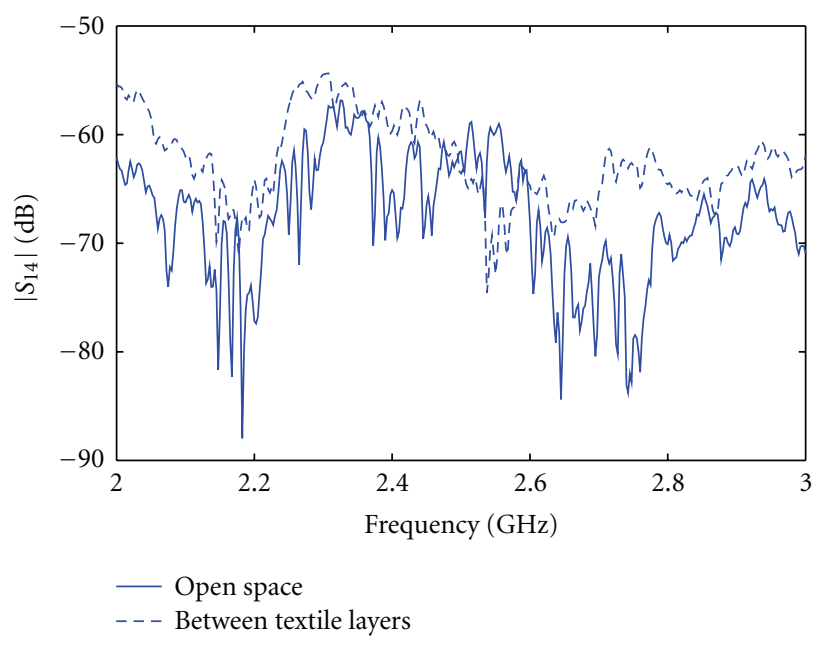

(f) Bent: mutual coupling between port 1 and 4

FIGURE 22: Mutual coupling between port 1 of the array and the other three ports, when the array is in planar and bent state.

a substrate for a textile antenna array to integrate into a garment, the MR value of the textile material must be taken into account. Consequently, a comprehensive study of the performance of the array system was carried out for the textile implementation on the Azzurri foam substrate only. Measurements of the reflection coefficient in the $2.45 \mathrm{GHz}$
ISM band demonstrate a large bandwidth, providing full coverage of the ISM band, both when the array system is operating in free space and when it is deployed on a human body. Simulations and measurements of the gain patterns in free space and on-body show very good correspondence, demonstrating that the human body does not degrade the 
performance of the textile antenna system. Injecting an input power of $0.5 \mathrm{~W}$ at each port of the array, the calculated SAR values averaged in $10 \mathrm{-g}$ tissue show that the textile antenna array respects the ICNIRP and FCC guidelines for general public exposure. Next, the mutual coupling between the four ports of the array was measured for varying $\mathrm{rH}$ levels, to check the sensitivity of the array to environmental changes. Moreover, a careful study of the mutual coupling between the four ports of the array was carried out with the array in planar and bent state, for different setups: in open space, between textile layers composing the firefighter suit, and inside the firefighter jacket. Thanks to the ETMPA topology of the individual patches and the rather large spacing of $3 / 4 \lambda$ between them, the mutual coupling is always low.

In summary, thanks to a careful design and a thorough study of mutual coupling effects, we managed to present a low-cost textile antenna array system functioning in the $2.45 \mathrm{GHz}$ ISM band, with low-cost steering capabilities, significantly higher gain than a single patch antenna, tolerant to being placed on the body and resistant to environmental changes, and resulting in reliable off-body communication links thanks to reduced fading.

\section{References}

[1] M. Klemm, I. Locher, and G. Tröster, "A novel circularly polarized textile antenna for wearable applications," in Proceedings of the 7th European Microwave Week, pp. 137-140, October 2004.

[2] P. Salonen and H. Hurme, "A novel fabric WLAN antenna for wearable applications," in Proceedings of the IEEE Antennas and Propagation Society International Symposium, pp. 700703, June 2003.

[3] E. K. Kaivanto, M. Berg, E. Salonen, and P. de Maagt, "Wearable circularly polarized antenna for personal satellite communication and navigation," IEEE Transactions on Antennas and Propagation, vol. 59, no. 12, pp. 4490-4496, 2011.

[4] G.-Y. Lee, D. Psychoudakis, C.-C. Chen, and J. L. Volakis, "Omnidirectional vest-mounted body-worn antenna system for UHF operation," IEEE Antennas and Wireless Propagation Letters, vol. 10, pp. 581-583, 2011.

[5] C. Hertleer, A. Tronquo, H. Rogier, and L. Van Langenhove, "The use of textile materials to design wearable microstrip patch antennas," Textile Research Journal, vol. 78, no. 8, pp. 651-658, 2008.

[6] C. Hertleer, H. Rogier, L. Vallozzi, and L. Van Langenhove, "A textile antenna for off-body communication integrated into protective clothing for firefighters," IEEE Transactions on Antennas and Propagation, vol. 57, no. 4, pp. 919-925, 2009.

[7] J. Vicente and A. A. Moreira, "Electro-textile printed slot antenna over finite EBG structure," in International Workshop on Antenna Technology (iWAT '10), pp. 1-4, March 2010.

[8] Q. Bai and R. Langley, "Wearable EBG antenna bending and crumpling," in Proceedings of the Loughborough Antennas and Propagation Conference (LAPC '09), pp. 201-204, Loughborough, UK, November 2009.

[9] L. Vallozzi, P. Van Torre, C. Hertleer, H. Rogier, M. Moeneclae$y$, and J. Verhaevert, "Wireless communication for firefighters using dual-polarized textile antennas integrated in their garment," IEEE Transactions on Antennas and Propagation, vol. 58, no. 4, pp. 1357-1368, 2010.
[10] L. Vallozzi and H. Rogier, "Radiation characteristics of a textile antenna designed for apparel application," in Proceedings of the IEEE Symposium for Space Applications of Wireless and RFID (SWIRF'07), May 2007.

[11] S. Zhu and R. Langley, "Dual-band wearable textile antenna on an EBG substrate," IEEE Transactions on Antennas and Propagation, vol. 57, no. 4, pp. 926-935, 2009.

[12] C. Du, S. Zhong, L. Yao, and Y. Qiu, “Textile microstrip array antenna on three-dimensional orthogonal woven composite," in Proceedings of the 4th European Conference on Antennas and Propagation (EuCAP'10), pp. 1-2, April 2010.

[13] C. Du, S. Zhong, L. Yao, and Y. Qiu, "Textile microstrip twoelement array antenna on 3D orthogonal woven composite," in Proceedings of the International Symposium on Signals, Systems and Electronics (ISSSE '10), vol. 2, pp. 1-2, September 2010.

[14] ICNIRP, "Guidelines for limiting exposure to time-varying electric, magnetic, and electromagnetic fields," Health Physics, vol. 74, no. 4, pp. 494-522, 1998.

[15] FCC, "Evaluating Compliance with FCC Guidelines for $\mathrm{Hu}$ man Exposure to Radiofrequency Electromagnetic Fields," Supplement C to OET Bulletin 65, Washington, DC, USA, 2001.

[16] http://www.lioninternational.fr/index.php.

[17] Y. Siddiqui Jawad and G. Debatosh, "Applications of triangular microstrip patch: circuit elements to modern wireless antennas," Mikrotalasna Revija, vol. 13, no. 1, pp. 8-11, 2007.

[18] S. Sadat Karimabadi, Y. Mohsenzadeh, A. Reza Attari, and S. Mahdi, "Bandwidth enhancement of single-feed circularly polarized equilateral triangular microstrip antenna," in Proceedings of the Progress in Electromagnetic Research Symposium, pp. 147-150, Hangzhou, China, March 2008.

[19] C. Tang, J. Lu, and K. Wong, "Circularly polarised equilateraltriangular microstrip antenna with truncated tip," Electronics Letters, vol. 34, no. 13, pp. 1277-1278, 1998.

[20] C. Hertleer, A. Van Laere, H. Rogier, and L. Van Langenhove, "Influence of relative humidity on textile antenna performance," Textile Research Journal, vol. 80, no. 2, pp. 177-183, 2010.

[21] F. Declercq, Characterization and design strategies for active textile antennas [Ph.D. thesis], Ghent University, 2011.

[22] F. Declercq, I. Couckuyt, H. Rogier, and T. Dhaene, "Complex permittivity characterization of textile materials by means of surrogate modelling," in Proceedings of the IEEE International Symposium Antennas and Propagation and CNCUSNC/ URSI Radio Science Meeting, July 2010.

[23] M. Klemm, I. Z. Kovcs, G. F. Pedersen, and G. Troster, "Novel small-size directional antenna for UWB WBAN/WPAN applications," IEEE Transactions on Antennas and Propagation, vol. 53, no. 12, pp. 3884-3896, 2005.

[24] C. Gabriel, S. Gabriel, and E. Corthout, "The dielectric properties of biological tissues: I. Literature survey," Physics in Medicine and Biology, vol. 41, no. 11, pp. 2231-2249, 1996.

[25] S. Gabriel, R. W. Lau, and C. Gabriel, "The dielectric properties of biological tissues: II. Measurements in the frequency range $10 \mathrm{~Hz}$ to $20 \mathrm{GHz}$," Physics in Medicine and Biology, vol. 41, no. 11, pp. 2251-2269, 1996.

[26] C. Gabriel, S. Gabriel, and E. Corthout, "The dielectric properties of biological tissues: III. Parametric models for the dielectric spectrum of tissues," Physics in Medicine and Biology, vol. 41, no. 11, pp. 2271-2293, 1996. 

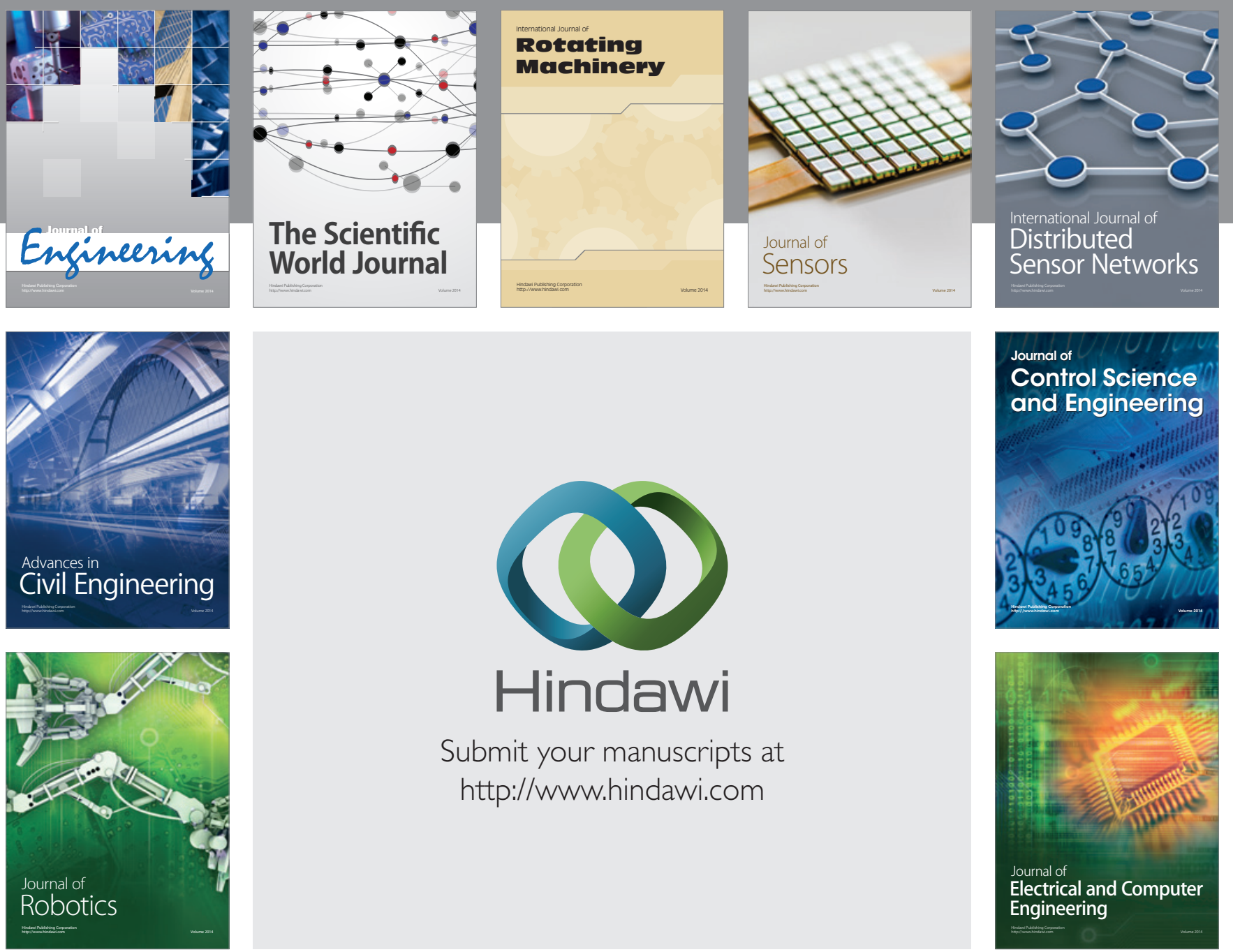

Submit your manuscripts at

http://www.hindawi.com
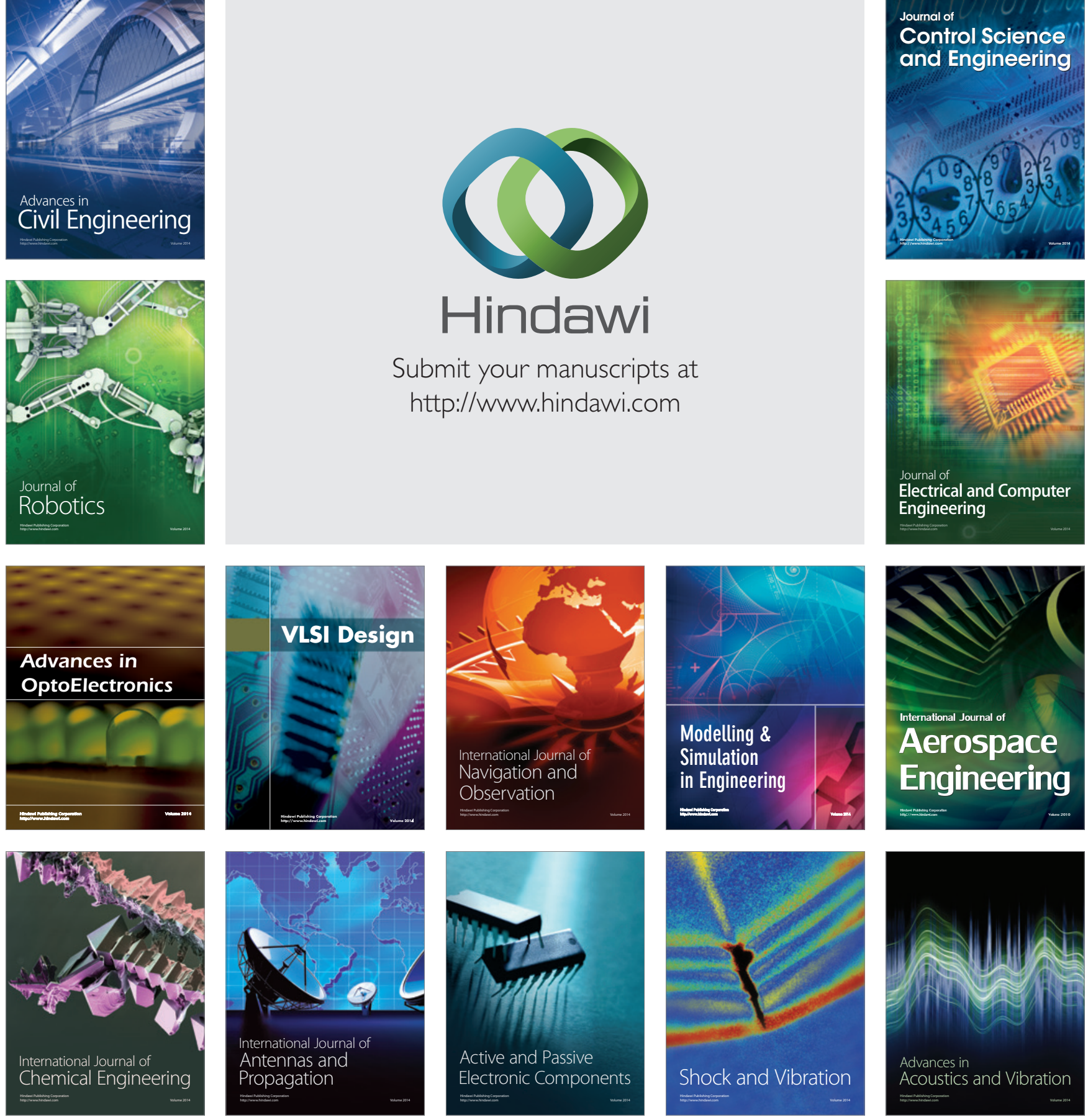